Recibido 05 de Enero, 2019 - Aceptado 05 de Febrero, 2019

\title{
Motivación y conductas de liderazgo en una poblacion militar de Arequipa
}

\section{Motivation and leadership behaviors in a military population of Arequipa}

\author{
Julio Abarca
}

\section{RESUMEN}

El objetivo de la investigación fue relacionar la motivación para liderar y las conductas del líder, en población militar en Arequipa. Se evaluó a 36 Oficiales, 25 varones y 11 mujeres, con personal a cargo. Se utilizó el cuestionario de motivación para liderar y el cuestionario de conductas del líder. Los resultados obtenidos por el análisis estadístico muestran que sí existe una relación significativa entre la motivación para liderar y las conductas del líder, además, existen relaciones significativas entre la motivación intrínseca para liderar y las conductas orientadas hacia la tarea, así como entre la motivación social normativa para liderar y las conductas orientadas hacia el cambio, sin embargo, no se encontró relación significativa entre la motivación extrínseca para liderar y las conductas orientadas hacia las relaciones. Se enfatiza la necesidad de la mejora continua y revalorizar aún más el liderazgo como una herramienta de gestión estratégica en las organizaciones.

Palabras clave: Líder, Liderazgo, Conductas del Líder, Motivación para Liderar.

\section{ABSTRACT}

The objective of the research was to relate the motivation to lead and the leader's behavior, in a military population in Arequipa. 36 officers, 25 men and 11 women were evaluated, with personnel in charge. The motivation to lead questionnaire and the leader behavior questionnaire were used. The results obtained by the statistical analysis show that there is a significant relationship between the motivation to lead and the behavior of the leader, in addition, there are significant relationships between intrinsic motivation to lead and task-oriented behaviors, as well as between social motivation norms to lead and behaviors oriented toward change, however, no significant relationship was found between extrinsic motivation to lead and behaviors oriented toward relationships. Emphasis is placed on the need for continuous improvement and to revalue leadership even further as a strategic management tool in Organizations.

Keywords: Leader, Leadership, Behavior of Leader, Motivation for Leading.

1. Psicólogo por la Universidad Nacional de San Agustín de Arequipa (UNSA), Maestro en Ciencias: Administración (MBA) con mención en Gerencia de Recursos Humanos, Egresado de la Segunda Especialidad de Promoción de la Salud y Terapia Familiar de la UNSA.

Docente a Tiempo Completo de la UAP - Filial Arequipa. Email: julioabar@gmail.com 


\section{INTRODUCCIÒN}

Actualmente, los líderes se encuentran en muchas partes y son diferentes a los que antes conceptualizábamos como tales porque en todas las organizaciones están sometidos a múltiples desafíos.

Existen resultados de investigaciones que muestran la presencia de una fuerte asociación entre los intercambios de alta calidad y la obtención de niveles altos de productividad, entre líderes y seguidores, así como también, un mayor nivel de satisfacción en el trabajo (Seers y Graen, 1984).

Eagly y Johnson (1990) realizaron un meta-análisis demostrando que las mujeres estaban ligeramente más orientadas hacia las conductas de relaciones personales que los hombres y no existían diferencias en el estilo de orientación a la tarea. Y que tanto los hombres como las mujeres líderes reflejaban un estilo de liderazgo orientado hacia la tarea cuando desempeñaban una actividad considerada congruente con su género.

A finales de los años ochenta y principios de los noventa los estudios de Lord, Foti y De Vader (1984); y Lord y Maher (1991) desde la perspectiva del procesamiento de la información resultaron en una línea de investigaciones de gran utilidad para el estudio del liderazgo en las organizaciones.

Lord y Maher (1991) sostienen que el liderazgo es fundamentalmente un proceso atributivo resultado de un proceso de percepción social, siendo la esencia del mismo el ser percibido como líder por los otros.

En esos estudios implican un resurgimiento del interés en el estudio de los rasgos de personalidad de los líderes pero poniendo énfasis en el papel de los prototipos cognitivos idiosincráticos que se forman en la mente de los seguidores respecto de líderes. Más específicamente, se han focalizado en la influencia que generan las expectativas previas y los prototipos internaliza- dos, sostenidos por los subordinados, en el nivel de efectividad que se percibe respecto de líderes actuales (Lord, Foti y De Vader, 1984; Lord y Maher, 1991).

El marco teórico de la Teoría del Intercambio (LMX, leader member exchanges) constituye otra línea de investigación en el estudio del liderazgo de gran difusión y con gran soporte empírico (Graen y Uhl-Bien, 1995).

Schartz-Kenney y Blascovich (1996) sugieren que los miembros de un grupo de trabajo desarrollan, a través de procesos de socialización y experiencias pasadas con líderes, una serie de teorías implícitas acerca del liderazgo (TILs) (citado por Engle y Lord, 1997).

La relación entre TILS y LMX no es recíproca. Resulta importante el estudio de ambos constructos desde el punto de vista de los seguidores, ya que los líderes no evalúan a los subordinados sobre la base de prototipos ideales sino en virtud de teorías implícitas relacionadas con la performance efectiva de dichos seguidores (Engle y Lord, 1997).

Asimismo, los líderes como los subordinados, poseen un guión o estereotipo sobre cuáles son las conductas esperadas de una persona para ser considerada líder, según afirman Wofford, Godwin y Wittington en 1998 (citado por Castro Solano, Lupano Perugini, Benatuil y Nader, 2007).

De acuerdo a Martínez y García (1999) ocupar un puesto de supervisión promueve mayor motivación intrínseca, dado a que se cuenta con mayor preparación académica, más experiencia laboral y mayor libertad en la toma de decisiones. Además, afirman que existen variables tales como el género, el nivel jerárquico, la edad, el tipo de organización, entre otros, que pueden afectar el nivel de motivación de un trabajador.

El estudio de la motivación para liderar o Motivation to Lead (MTL) se basa fundamentalmente en las investigaciones de dos autores: Chan y 
Drasgow (2001), quienes consideran que el liderazgo es un fenómeno sumamente complejo $y$, por lo tanto, es multideterminado, esto implica que no puede ser explicado solamente por un rasgo psicológico único o algo en particular. Consideran la MTL como un constructo específico que afecta las decisiones de los líderes tanto durante su entrenamiento como en el ejercicio de su rol en específico. Además, consideran las diferencias individuales al asumir la formación en liderazgo, los roles y responsabilidades que afectan la intensidad del esfuerzo y la persistencia como líder. Proponen tres dimensiones: motivación intrínseca, motivación extrínseca, y motivación social normativa.

El estudio de las conductas del líder, se ha dado desde que comenzó a abordarse científicamente el liderazgo, y existen diferentes teóricos que han sostenido muchas teorías por varias décadas. Yukl (2002) propone un modelo de las conductas de líder que da lugar a distintas versiones de un cuestionario basado en tres factores previstos. Yukl, Gordon y Taber (2002) confirman ello empíricamente en distintas muestras, a distintos niveles de análisis, individual y de equipo, y para diferentes contextos organizacionales.

El paradigma dominante en el estudio del liderazgo evolucionó desde modelos más estáticos basados en los rasgos psicológicos de los líderes a modelos situacionales que toman en cuenta las variables del ambiente y de la persona (Yukl, 2002).

Cuadrado, Molero y Navas (2003) exponen que cuando las mujeres adoptan un estilo orientado hacia las tareas contribuyen a que se sientan satisfechas con el modo en que dirigen, mientras que para los hombres, éste estilo predice la eficacia de su departamento. Cabe añadir, que aunque no se encontraron diferencias estadísticamente significativas entre hombres y mujeres en cuanto a las conductas orientadas hacia las relaciones, las mujeres obtuvieron el promedio más alto en este tipo de conductas.
Según Yukl (2004) el líder manifiesta abiertamente una serie de conductas observables e identificables que se basan fundamentalmente en: Conductas orientadas hacia la tarea, conductas orientadas hacia las relaciones, y conductas orientadas hacia el cambio.

El campo compuesto por el liderazgo es muy heterogéneo e incluye un amplio panorama de especulaciones, enunciaciones y apreciaciones. $\mathrm{Su}$ estudio se ha abordado desde diferentes disciplinas como la historia, la teoría psicodinámica, la teoría del desarrollo organizacional y la sociología (Kroeck, Lowe \& Brown, 2004).

El liderazgo puede ser definido como un proceso natural de influencia que ocurre entre un líder y sus seguidores. Este proceso de influencia puede ser explicado a partir de: características y conductas del líder, percepciones y atribuciones por parte de los seguidores y/o por el contexto en el cuál ocurre dicho proceso (Antonakis, Cianciolo \& Sternberg, 2004).

Epitropaki y Martin (2005) sostienen que cualquier discrepancia hallada entre el prototipo ideal y el real afecta la impresión que los seguidores se forman acerca de sus líderes. Estos prototipos son altamente idiosincrásicos, por lo cual es esperable encontrar diferentes prototipos de líderes en diferentes grupos sociales y poblaciones. Y que en la medida en que los seguidores reconozcan una coincidencia entre el prototipo de líder internalizado y las conductas efectivas de sus superiores, los intercambios líder-seguidor tienden a ser de alta calidad.

Se observa que existen diferencias en la percepción de las conductas de los líderes según el tipo de puesto y según el tipo de cultura organizacional, mientras que no se observan diferencias según el tipo de población, sea contexto civil o militar, donde se ejerce el liderazgo (Lupano Perugini; Blanco y Castro Solano, 2006).

A mayor discrepancia entre los atributos del líder percibido y los del líder ideal la relación con el 
superior es de peor calidad. En el mismo caso, las TILs moderan la relación que se establece con el jefe, si el líder no coincide con el prototipo que tienen en mente los seguidores, la relación también se ve afectada. No existe relación directa entre los prototipos de líder internalizados y la discrepancia entre el líder real/ideal (Castro Solano y Lupano Perugini, 2007).

Castro Solano (2008) en un estudio llevado a cabo en población militar, encontró que los militares no consideran que el bienestar y la satisfacción en el trabajo se expliquen a partir de la calidad de los intercambios establecidos entre un líder y sus subordinados.

\section{MATERIALES Y MÉTODOS}

\section{Participantes}

Los participantes fueron seleccionados según un muestreo por conveniencia, considerando que es el conjunto de todos los casos que concuerdan con determinadas especificaciones; el universo o población de nuestro estudio está representado por todos los Oficiales del Cuartel Mariano Bustamante de la $3^{\text {a }}$ Brigada de Servicios de la III División del Ejército (DE), Arequipa, 2015; con excepción del Coronel, en el siguiente número y grado militar: 10 Teniente Coronel, 8 Mayor, 6 Capitán, 4 Teniente, 8 Sub Teniente. En relación a algunas características sociodemográficas, consideramos respecto al sexo, se contó con 25 oficiales de sexo masculino y 11 oficiales de sexo femenino; en relación a la edad, se contó con 4 categorías, de 18 a 27 años (4 oficiales), de 28 a 37 años (10 oficiales), de 38 a 47 años (12 oficiales) y de 48 a más años (10 oficiales).

Asimismo, se consideró el grado de instrucción, encontrándose con postgrado (15 oficiales) y de nivel superior ( 21 oficiales), en referencia a la antigüedad en el tiempo de servicio en el cargo, se detalló 4 categorías, menor de 2 años (9 oficiales), de 2 a 5 años (20 oficiales), de 6 a 9 años (3 oficiales) y de más de 9 años (4 oficiales).
Se determinó la muestra, cuyo tamaño es conforme al proporcionado al cuadro de Oficiales en actividad. Por lo que en la presente investigación se ha evaluado a todos los Oficiales del Ejército del Perú que se encuentran en las instalaciones del Cuartel Mariano Bustamante que es sede de la $3^{\text {a }}$ Brigada de Servicios. El tamaño muestral, corresponde a toda la muestra de estudio para que la muestra sea representativa, es decir, toda la población, con excepción del Coronel por razones de estrategia militar.

\section{Escalas de Medición}

La escala de medición de las variables planteadas en el instrumento de recolección de datos, corresponde a la escala de Likert (1965), que viene a ser una medición ordinal que ha sido probada y estandarizada en múltiples ocasiones y se puede emplear como escalas de respuesta a preguntas o afirmaciones que mide juicios de valor sobre determinadas variables mediante percepciones de las unidades de estudio.

En los cuestionarios utilizados, las respuestas están enmarcadas dentro de una escala que va de 1 a 5, donde 1 representa a totalmente en desacuerdo y 5 representa a totalmente de acuerdo.

\section{Cuestionario de conductas del líder (CONLID)}

Es un cuestionario cuyo objetivo es evaluar los diferentes tipos de conductas percibidas en los líderes. El instrumento final consta de 18 ítems que se responden de manera autoadministrable.

Los ítems consisten en una serie de frases que describen posibles conductas de los líderes. Los sujetos deben responder, sobre la base de un formato de respuesta tipo Likert con 5 opciones, en qué grado dichas frases describen las conductas que ellos perciben en sí mismos. Siendo su puntaje mínimo 3 y su puntaje máximo 30 por cada indicador. Posee percentiles y niveles alto, medio y bajo, según sea su puntaje (Castro Solano, Nader y Lupano Perugini, 2005). 


\section{Cuestionario de motivación para liderar (PO- TENLID)}

Es un cuestionario cuyo objetivo es evaluar las motivaciones que presentan las personas para liderar. El instrumento consta de 9 ítems que se responden de manera autoadministrable. Se contestan en formato tipo Likert con 5 opciones de respuesta, se responde aproximadamente en 5 minutos. Siendo su puntaje mínimo 3 y su puntaje máximo 15 por cada indicador. Posee percentiles y niveles alto, medio y bajo (Castro Solano, 2005).

\section{Procedimiento}

Se contactó con el Coronel y con el Comandante Jefe del Estado Mayor y los responsables del Cuartel Mariano Bustamante para explicar el contenido y desarrollo de la investigación, se solicitó la autorización respectiva, se logró la misma. Con posterioridad, se produjo una reunión informativa con los jefes y responsables de las Unidades del mencionado Cuartel, quienes informaron a todos los Oficiales, al mismo tiempo que se les citaba la evaluación. Posteriormente, se les aplicó los instrumentos de la investigación. Se comunicó a los participantes sobre el procedimiento y se garantizó en todo momento su anonimato.

El trabajo de campo se llevó a cabo con el apoyo de asistentes previamente capacitados y bajo la supervisión del autor de la tesis. La aplicación de los instrumentos se hicieron in situ, en el Cuartel Mariano Bustamante que es sede de la $3^{\text {a }}$ Brigada de Servicios (Gran Unidad Militar) y en sus Unidades, mediante un horario de la ejecución de los cuestionarios que fue dentro de la jornada laboral de los Oficiales, y siendo atendidos por el Oficial de mayor jerarquía.

El estudio corresponde a un diseño no experimental de tipo transversal, con nivel descriptivo, cuyo proceso de tratamiento de la información recogida se sometió a los procedimientos de correlación, tabulación, interpretación y conclusiones.

\section{El proceso, presentó las siguientes etapas:}

1ra. etapa: Estimación de la población o universo de las unidades de estudio y determinación de la muestra correspondiente.

2da. etapa: Aplicación de pruebas de contenido del instrumento.

3ra. etapa: Desarrollo de dinámicas de sensibilización y capacitación del personal de apoyo, para el acopio de información de campo, así como la toma de datos.

4ta. etapa: Tabulación y procesamiento de información.

Los procedimientos estadísticos aplicados, fueron en función del tipo, diseño y nivel de investigación, entre los que podemos mencionar el procesamiento de información y tabulación que se realizó en Excel y en el paquete estadístico SPSS. Los resultados se manejaron a nivel de la estadística descriptiva, tomando en cuenta la calificación, los percentiles, los niveles, las frecuencias relativas y acumuladas, etc. En cuanto a las pruebas estadísticas, se trabajó con el coeficiente de Alfa de Cronbach para medir la fiabilidad, validez y factibilidad del instrumento, asimismo, en el proceso correlacional se apeló al Coeficiente de Correlación de Pearson para comprobar las Correlaciones Estadísticas.

\section{RESULTADOS}

Se ha considerado un número de elementos total de 27 ítems de evaluación, en consecuencia, de los 9 y 18 ítems iniciales, de los instrumentos POTENLID y CONLID, respectivamente, quedan los mismos, es decir, que según el coeficiente Alfa de Cronbach se obtuvo un valor de 0,951 que es válido. Para la investigación los ítems demuestran capacidad para medir lo que pretenden medir y no otros aspectos de los pretendidos y como es de suponer los resultados finales y los análisis pertinentes se harán a partir de éstos ítems que demostraron fiabilidad. 
Tabla No 1. Correlación entre las variables de la Motivación para liderar y de las Conductas del líder

\begin{tabular}{|c|c|c|c|}
\hline & & $\begin{array}{l}\text { MOTIVACIÒN } \\
\text { PARA } \\
\text { LIDERAR }\end{array}$ & $\begin{array}{l}\text { CONDUCTAS } \\
\text { DEL LIDER }\end{array}$ \\
\hline \multirow{3}{*}{$\begin{array}{l}\text { MOTIVACION } \\
\text { PARA } \\
\text { LIDERAR }\end{array}$} & $\begin{array}{l}\text { Correlación } \\
\text { de Pearson }\end{array}$ & 1 & $.744^{* *}$ \\
\hline & $\begin{array}{l}\text { Sig. } \\
\text { (bilateral) }\end{array}$ & & .000 \\
\hline & $\mathrm{N}$ & 36 & 36 \\
\hline \multirow{3}{*}{$\begin{array}{l}\text { CONDUCTAS } \\
\text { DEL LIDER }\end{array}$} & $\begin{array}{l}\text { Correlación } \\
\text { de Pearson }\end{array}$ & $.744^{* *}$ & 1 \\
\hline & $\begin{array}{l}\text { Sig. } \\
\text { (bilateral) }\end{array}$ & .000 & \\
\hline & $\mathrm{N}$ & 36 & 36 \\
\hline
\end{tabular}

La Tabla 1, brinda información sistemática, teórica y práctica en relación a lo más importante en lo operativo de las funciones asumidas por los Oficiales en su ejercicio de liderazgo, donde se correlacionan de forma muy significativa los indicadores en estudio, es decir, que la motivación para liderar se relaciona de forma excelente con las conductas del líder.

Se ha determinado, en primer lugar, que sí existe un grado de relación significativo entre la motivación para liderar y las conductas del líder; comprobándose que existe una predominancia mientras más motivación se presente repercutirá directamente sobre las conductas que favorezcamos en el futuro.

Tabla No 2. Correlación entre indicadores de la Motivación para liderar y de las Conductas del líder

\begin{tabular}{|c|c|c|c|c|c|c|c|}
\hline & & MOT INTR & MOT EXTR & MOT SOCIAL & C. TAREA & C. RELACIONES & C. CAMBIO \\
\hline \multirow{3}{*}{ MOT INTR } & $\begin{array}{l}\text { Correlación } \\
\text { de Pearson }\end{array}$ & 1 & $-.439^{* *}$ & $.607^{*+}$ & $.782^{* *}$ & $.801^{* *}$ & $.752^{* *}$ \\
\hline & $\begin{array}{c}\text { Sig. } \\
\text { (bilateral) }\end{array}$ & & .007 & .000 & .000 & .000 & .000 \\
\hline & $\mathrm{N}$ & 36 & 36 & 36 & 36 & 36 & 36 \\
\hline \multirow{3}{*}{ MOT EXTR } & $\begin{array}{l}\text { Correlación } \\
\text { de Pearson }\end{array}$ & $-.439^{* *}$ & 1 & -.164 & -.261 & $-.333^{*}$ & -.133 \\
\hline & $\begin{array}{c}\text { Sig. } \\
\text { (bilateral) }\end{array}$ & .007 & & .339 & .125 & .047 & .438 \\
\hline & $\mathrm{N}$ & 36 & 36 & 36 & 36 & 36 & 36 \\
\hline \multirow{3}{*}{ MOT SOCIAL } & $\begin{array}{l}\text { Correlación } \\
\text { de Pearson }\end{array}$ & $.607^{* *}$ & -.164 & 1 & $.670^{* *}$ & $.637^{*+}$ & $.684^{* *}$ \\
\hline & $\begin{array}{c}\text { Sig. } \\
\text { (bilateral) }\end{array}$ & .000 & .339 & & .000 & .000 & .000 \\
\hline & $\mathrm{N}$ & 36 & 36 & 36 & 36 & 36 & 36 \\
\hline \multirow{3}{*}{ C. TAREA } & $\begin{array}{l}\text { Correlación } \\
\text { de Pearson }\end{array}$ & $.782^{* *}$ & -.261 & $.670^{* *}$ & 1 & $.963^{* *}$ & $.934^{* *}$ \\
\hline & $\begin{array}{c}\text { Sig. } \\
\text { (bilateral) }\end{array}$ & .000 & .125 & .000 & & .000 & .000 \\
\hline & N & 36 & 36 & 36 & 36 & 36 & 36 \\
\hline \multirow{3}{*}{ C. RELACIONES } & $\begin{array}{l}\text { Correlación } \\
\text { de Pearson }\end{array}$ & $.801^{* *}$ & $-.333^{*}$ & $.637^{*}$ & $.963^{* *}$ & 1 & $.927^{* *}$ \\
\hline & $\begin{array}{c}\text { Sig. } \\
\text { (bilateral) }\end{array}$ & .000 & .047 & .000 & .000 & & .000 \\
\hline & $\mathrm{N}$ & 36 & 36 & 36 & 36 & 36 & 36 \\
\hline \multirow{3}{*}{ C. CAMBIO } & $\begin{array}{l}\text { Correlación } \\
\text { de Pearson }\end{array}$ & $.752^{* *}$ & -.133 & $.684^{* *}$ & $.934^{* *}$ & $.927^{* *}$ & 1 \\
\hline & $\begin{array}{c}\text { Sig. } \\
\text { (bilateral) }\end{array}$ & .000 & .438 & .000 & .000 & .000 & \\
\hline & $\mathrm{N}$ & 36 & 36 & 36 & 36 & 36 & 36 \\
\hline
\end{tabular}


La Tabla 2, demuestra que las variables del estudio se correlacionan de forma muy significativa con excepción de la motivación extrínseca y de todas sus relaciones.

Es decir, por presentar una motivación social normativa es posible presentar conductas orientadas hacia el cambio, y viceversa; es el mismo caso de correlación para la aparición de la motivación intrínseca con las conductas orientadas hacia la tarea. Sin embargo, respecto a la motivación extrínseca y su relación con las conductas orientadas hacia las relaciones, no se comprueba nuestra hipótesis de investigación, aunque existe un valor no significativo que abre la posibilidad a realizar nuevas investigaciones sobre éste análisis en particular. Esto último es muy interesante puesto que significa que no importa cuán mayor motivación extrínseca se pueda introducir en diversos contextos los resultados respecto a liderazgo en cualquiera de sus variables serán negativos o inversos.

Por lo tanto, se ha determinado en segundo lugar que existe una relación muy significativa entre la motivación intrínseca para liderar y las conductas del líder orientadas hacia la tarea, por lo que se desprende la importancia de buscar de forma independiente y voluntaria ser líder para presentar inequívocamente comportamientos que guíen indefectiblemente al cumplimiento de tareas, actividades y funciones propias del ejercicio de los líderes actuales, además de establecer por medio de programas de entrenamiento para el liderazgo nuevos mecanismos para posibilitar que los líderes se motiven y actúen en este grado de correspondencia, motivados naturalmente y siempre dirigiéndose al cumplimiento de las tareas en primer término.

En tercer lugar, se ha definido que no existe relación significativa entre la motivación extrínseca para liderar y las conductas del líder orientadas hacia las relaciones, demostrándose que no existe ningún grado de relación entre éstos indicadores y que si además, si se intentara por algún método tratar de hacer que ocurra la presentación de beneficios para liderar en una persona elegida no presentará nunca comportamientos dirigidos a mejorar las relaciones sociales con otras personas, si no que se conseguirá todo lo contrario, de forma inversa o una respuesta de manera negativa.

Finalmente, en cuarto lugar se ha comprobado que sí existe un alto grado de relación significativa entre la motivación social normativa para liderar y las conductas del líder orientadas hacia el cambio, que nos demuestra que si bien hay una prevalencia positiva entre desear ser líder de otros procurando la justicia social y el presentar comportamientos sostenidos de completa facilidad para hacer cambios institucionales y sociales, por lo que existe la necesidad de potenciar estos conceptos y realizar mayores investigaciones respecto a los beneficios que representaría ello.

\section{DISCUSIÓN}

El propósito es comprobar o refutar el grado de relación entre la motivación para liderar y las conductas del líder, en base a sus indicadores, en personas que tengan a su cargo subordinados, como parte importante de su gestión. En general los modelos de estudio para abordar la problemática del liderazgo han pasado de unos ejes de análisis a otros ejes diferentes unos entre otros, lo que nos afirma Yukl (2002).

Para ello, la presente investigación dentro de los Paradigmas de Investigación en el Estudio de la Motivación se asoció al paradigma correlacional, ya que más que manipular variables se busca encontrar las relaciones existentes entre ellas, buscando responder las interrogantes planteadas a través de la interpretación de lo obtenido en la muestra.

La aplicación del análisis se realizó sobre una de las Brigadas de Oficiales más grande de la III División del Ejército, que es muy representativa de la República del Perú, incluso es considerada la División de Hierro, nombre que le dan debido a su fortaleza y excepcionalidad demostrada a 
través de la historia, que podrían algunos considerar como una cierta limitación de alcance, no obstante, servirá de aporte para futuras investigaciones, así como de ayuda a la sociedad organizada y empresarial.

Se ha podido concluir que la presente investigación ha identificado patrones de conducta que han ayudado a los Oficiales del Ejército, que se constituyen como líderes participantes en su organización, a tomar acciones estratégicas para reaccionar a los cambios constantes del entorno. Debido a que se han podido encontrar algunas similitudes y diferencias entre la teoría y la investigación se han podido mejorar conceptos importantes acerca del liderazgo, pero se ha dado inicio hacia nuevas investigaciones, respecto al liderazgo institucional (formalizado), liderazgo militar, liderazgo auto-percibido y gestión de personas y organizaciones.

Los indicadores identificados y que representan la variable independiente motivación para liderar corresponden a: motivación intrínseca, motivación extrínseca y motivación social normativa se han presentado en los participantes del estudio, en niveles altos y medios con valores superiores y en menor incidencia niveles bajos pero que han ocurrido debido a las especificaciones de las funciones de algunos Oficiales o la menor antigüedad en la Institución. Asimismo, los indicadores identificados y que representan para el estudio, a la variable dependiente conductas del líder corresponden a: conductas orientadas hacia las tareas, conductas orientadas hacia las relaciones y conductas orientadas hacia el cambio, que también se han dado mayoritariamente en niveles altos y luego medios, existiendo pocos casos en niveles bajos.

Las motivaciones y las conductas se encuentran alineadas de manera natural, lo cual se comprueba a través de investigaciones encontradas en la teoría, acerca de este hecho en múltiples poblaciones, empero, respecto al ejercicio práctico del liderazgo dada su verdadera complejidad no se presenta tal alineación (como indicaba la teoría en general), ni existe un determinismo cultural, ni histórico referente a la unión de las variables de estudios; y además, al estudiar sus indicadores la tarea se vuelve mucho más compleja en análisis y reflexión, ya que no concuerdan plenamente.

Los objetivos personales y organizacionales se alinean en la muestra de estudio debido a que los Oficiales y la alta dirección de la Institución pertenecen a un mismo grupo (ya que los Oficiales tienden a cumplir sus funciones y posteriormente ascienden hasta llegar a ser la parte más elevada de toda la organización).

Un factor que da garantías para ser un líder efectivo es concentrarse en el cumplimiento de las tareas asignadas, lo que les proporciona una capacidad de toma de decisión ágil y flexible, que evidentemente influye en el gobierno de la institución, y permite que ésta se adapte más fácilmente a los cambios del entorno.

Castro Solano (2008) afirmó que las relaciones interpersonales entre líder y subordinados no pueden garantizar ni bienestar ni satisfacción en el trabajo en población militar, con lo que se comprobaría que no se le otorga importancia a ser líder gracias a que tiene conductas orientadas hacia las relaciones y en alguna medida relación con ser motivado de manera externa, lo cual justificaría los resultados de la presente investigación respecto a las relaciones y correlaciones dadas en base a esos indicadores.

Además, respecto a la probabilidad que exista motivación social normativa que influencie para que se originen conductas orientadas hacia el cambio no existe relación ni correlación significativa entre tales indicadores, no obstante, existe un nivel positivo respecto a la predominancia de forma positiva lo cual en una mayor población o en un sector diferenciado al militar, tal vez el contexto empresarial, podría corroborarse y ver si en general existiría esta relación debido al estudio donde existe hoy en día mas interés en la innovación y el cambio. 
Un componente adicional en el que se comprueba la teoría del liderazgo desde una perspectiva conductual es el control de la información y el monitoreo de la misma producto del componente de confianza que se encuentra en organizaciones de tipo vertical.

Aunque no podríamos definir lo investigado por científicos entendidos en el tema, donde se afirma que una de las ventajas de la planificación y organización es la fluidez de la comunicación entre los miembros de las instituciones. No existe separación entre participación y control de la Institución. Por esta razón, los intereses de los líderes no se desenvuelven en estructuras formales de procedimientos, controles y códigos. Las relaciones estrechas, de confianza y de familiaridad en el sistema de control interno han permitido alcanzar en la Institución la posibilidad para desenvolverse de manera óptima. No obstante, están también conscientes que a medida que crezca el número de personas participantes, este mecanismo debe ser más estructurado para no perder esta ventaja.

La literatura considera que existe el peligro de perder la visión al concentrar distintos roles en las mismas personas y mantener relaciones plenamente no estructuradas. Con todo, la realidad muestra que el tipo de liderazgo compartido regula este peligro cuando están presentes más miembros de la Institución, ya que provee un marco de discusión y consenso. El sistema de liderazgo institucional utilizado en la muestra corresponde al modelo latino, en el cual se considera que el objetivo principal se encuentra en el beneficio no sólo de los accionistas, sino también de los grupos de interés que se relacionan con ella.

No existen procedimientos formales para la contratación de la alta dirección en la Institución, pues ellos son miembros de la organización desde grados inferiores y van en ascenso cada cierta cantidad de tiempo, y es cuestión de un gran despliegue de habilidades para que se integren paulatinamente a la Institución y, como tales, se espera que tomen esa posición. El contrato no formal que se establece con ellos es del tipo de relación de conducta, pues se considera que son contratos basados en que la organización maneje objetivos de bienestar social. Por otro lado, los contratos basados en incentivos de compensación se encuentran ausentes en la Institución estudiada ya que no son los determinantes. En las empresas, se observa que los medios de control externo como el sistema regulatorio y el mercado de productos y servicios, han influido de manera dominante en aquellas organizaciones.

Las acciones tomadas a partir de hitos históricos en la Institución son las que prevalecen a diferencia de las empresas donde normas temporales señaladas en la década de 1990 muestran la supervivencia de empresas familiares y otras organizaciones, lo que ha sido posible por una rápida adaptación al nuevo entorno en el que se desenvuelven, producto de un cambio dramático en las condiciones de control que se ha referido antes. Esto confirma la teoría acerca de la influencia de los controles externos en las empresas, y nos muestra lo contrario respecto a la Institución en estudio.

Existen organismos de gobierno corporativo formalmente establecidos como son el directorio y el consejo de administración dentro de las organizaciones empresariales. Sus funciones muchas veces se encuentran superpuestas debido a que las personas encargadas de esos roles cumplen otros simultáneamente. La informalidad en el tratamiento de dichos organismos muestra que en la mayoría de casos están constituidos sólo para el cumplimiento de un requisito legal y no sirve como herramienta de gestión.

La práctica encontrada en la Institución estudiada muestra que esta forma de acción no resulta una amenaza dado el libre acceso a la información. El tipo de directorio o personas a cargo de otras personas que se maneja es proactivo y de búsqueda de valor, debido a la concepción de la Institución como un espíritu de cuerpo, cuya permanencia se debe asegurar en el tiempo y no 
se imponen restricciones entre sus miembros de ningún tipo. Los organismos que se puedan crear para volver el liderazgo sostenible serían de mayor utilidad, en las cuales la propiedad no se encuentra concentrada.

El ejercicio de elaborar un protocolo de liderazgo militar no ha sido realizado en todas los Ejércitos debido a que no han sentido una necesidad, ni identificado una razón que lo exija; sin embargo, algunas grandes potencias del mundo han visto ese tema como importante y han logrado elaborar Manuales de Campaña con tal temática. En las que sí se ha realizado, no existe evidencia de que se trate de un documento vivo al que se realicen consultas repetidas o actualizaciones periódicas. La explicación a esta realidad se puede dar por el reducido número de líderes militares presentes a niveles mundial dados los contextos de paz que vivimos, así como por el hecho de que la Institución Militar estudiada se encuentra en un grado de desarrollo de nuevas generaciones.

Se ha empezado a tomar mayor interés en la inclusión de miembros líderes a todas las organizaciones, desde su búsqueda, reclutamiento, selección, formación y capacitación. Se considera que dichos integrantes permitirán una visión imparcial y externa de las situaciones que se enfrentan dentro de las organizaciones y mantendrán una postura no influenciada con el componente emocional propio del resto de participantes, por lo que desarrollaran y mejoraran los niveles de producción y satisfacción institucional. Esto es evidencia de la toma de consciencia de la sociedad al reconocer el componente emocional como factor que puede afectar y sesgar sus decisiones. Este podría ser el primer paso en el camino de la organización interna hacia un modelo de gestión más profesional en la Institución Militar.

Según los resultados obtenidos, los líderes militares resaltan la importancia de la unidad organizacional, la cual califican de influencia favorable y positiva tanto para los miembros de la organización como para los fines de la Nación. Esto con el objetivo de lograr resultados óptimos y la con- tinuidad de la mejora continua que se está emprendiendo en todas las instituciones nacionales. Por otro lado, se sostiene que los valores cultivados dentro de las familias trascienden directamente sobre los valores de la organización. Éstos actúan como base para la continuidad en las siguientes generaciones y son determinantes para desarrollar un ambiente de confianza y establecer una cultura institucional óptima.

Según los resultados, los líderes de las organizaciones se preocupan más en la motivación intrínseca y en el cumplir las tareas encomendadas, estableciendo relaciones duraderas con los diferentes grupos de interés, lo que es una ventaja, que busca mantener relaciones a largo plazo y consolidar lazos de confraternidad que perduren en el tiempo, de una forma natural sin necesidad de motivos externos o secundarios.

La participación activa de los líderes en la dirección de las organizaciones es muy importante por el hecho de querer asumir grandes responsabilidades sin presión social, ni influencia de terceros. Ahora bien, en los casos en que el líder predominante se encuentra aún presente, se identifica una dominante influencia en las decisiones de otros líderes. En los casos en que el líder se encuentra más cerca de la gestión, lo que predomina es un liderazgo compartido entre los miembros más cercanos al líder y el propio líder. Por ello, algunos estudiosos consideran uno de los errores más comunes en la gestión de las organizaciones el retraso del traspaso de poder a las siguientes generaciones de líderes en el momento oportuno.

Entonces, la organización puede ser afectada en su gestión, ya que retrasa a su vez los nuevos planteamientos que se hacen necesarios frente a nuevos entornos. Desde un punto de vista particular se aprecia una excesiva confianza en la falta de necesidad de desarrollar mecanismos de prevención que faciliten el traspaso del poder y aseguren la permanencia del liderazgo en el futuro. Los miembros de las organizaciones se preocupan por los problemas de corto plazo y no 
prevén posibles conflictos que puedan generarse en el futuro, y en ese preciso momento se da importancia a los comportamientos de los líderes. La visión de largo plazo se renueva y prolonga, lo cual conlleva a nuevas inversiones y proyectos. Es determinante la posibilidad de adaptación y reenfoque de las estrategias según la experiencia de los líderes y puede considerarse como un factor clave de éxito organizacional.

Sobre las perspectivas para el futuro, el deseo de mantener el liderazgo entre los líderes de determinado entorno es compartido por todos en su organización. No obstante, la consideración sobre si los miembros de las futuras generaciones querrán tomar el mando, o inclusive si estarán capacitados, es una duda que hace que probable el hecho de implementar la colaboración de terceros. Se tiene muy claro que el mantener una organización de la misma manera como se lleva en la actualidad, o como se ha venido llevando, se hace más difícil a medida que nuestra sociedad crece y los miembros aumentan, lo que lleva a proyectar una natural profesionalización y organización de la gestión de todas las organizaciones que deseen ser mejores y en especial la Institución estudiada.

Se considera que debe existir un organismo que tome la responsabilidad de difundir los estudios acerca del liderazgo (público y privado) y faciliten la puesta en práctica de las recomendaciones que éste y otros estudios puedan aportar. Es por ello que se recomienda que las instituciones académicas tomen un rol activo, donde se estructuren programas educativos que desarrollen los temas más importantes como el uso, la ventaja del liderazgo compartido, la renovación de visión a largo plazo con la adecuada y planificada incorporación de las nuevas generaciones. De esta manera se proporcionará un marco académico donde se identifiquen y promuevan las habilidades blandas en la Gestión del Talento Humano, con la finalidad de llenar los vacíos y ofrecer este apoyo a la consolidación de organizaciones de éxito que beneficiará a la economía de todo el país.

El estudio es importante porque es uno de los primeros en investigar en el ámbito militar peruano la relación entre la motivación y las conductas presentes en el liderazgo. El propósito es servir de base para que las organizaciones tengan un mejor desempeño en base a sus líderes, lo que permitirá extender la permanencia en el tiempo de líderes en las organizaciones, beneficiando a la sociedad en general. También pretende servir de guía para jefes y miembros de instituciones en diferentes entornos. Para ello, se recomienda replicar el estudio utilizando diferentes muestras.

A través de la investigación realizada se ha concluido que, si bien existe interés por parte de todos en tener líderes alrededor del lugar de trabajo, se han encontrado en muchos casos que también existe falta de conocimiento acerca de la importancia del liderazgo, y este liderazgo es una herramienta de gestión corporativa estratégica.

Tomando como ventaja la característica principal de la ágil toma de decisiones debido a la cercanía de las relaciones interpersonales de la alta dirección y el directorio principal. Es notoria la necesidad de generar estudios en las áreas de liderazgo, que puedan servir de guía y asesoría en la evolución y desarrollo de las organizaciones. Este estudio fue realizado haciendo uso de una muestra promedio y en un sector militar representativo, por lo cual sus resultados son generalizables; y se recomienda realizar investigaciones en otros sectores.

Se exhorta también realizar estudios cuantitativos en diferentes sectores para poder identificar tendencias de mayor profundidad y alcance. Sería importante determinar si factores como el contexto y la cultura de la muestra explican la no confirmación de algunas hipótesis. Por lo que se sugiere estudios longitudinales y en otros entornos sociales. Si bien se ha considerado que se ha generado un avance al presentar las relaciones entre motivación y conducta, se considera que existen preguntas sin responder, y por ello se recomienda expandir el conocimiento dentro del área de estudio. 


\section{REFERENCIAS BIBLIOGRÁFICAS}

Camps, V.; Pérez, J. y Martínez, M. (2010). Comparación por género de los estilos de liderazgo en una muestra de gerenciales en Puerto Rico. Revista Puertorriqueña de Psicología, (21), pp. 113-132.

Castro Solano, A., Lupano Perugini, M., Benatuil, D. y Nader, M. (2007). Teoría y Evaluación del Liderazgo. Argentina: Editorial Paidós.

Castro Solano, A. (2005). Cuestionario de Motivación para Liderar POTENLID. Argentina: Editorial Paidós.

Castro Solano, A.; Nader, M. y Lupano Perugini, M. (2005). Cuestionario de Conductas del Líder CONLID-Forma A. Argentina: Editorial Paidós.

Castro Solano, A. y Lupano Perugini, M. (2007). Teorías implícitas del liderazgo y la calidad de la relación entre líder y seguidor. Boletín de Psicología, (89), 7-28.

Castro Solano, A. (2008). Teorías implícitas del liderazgo, calidad de la relación entre líder y seguidor y satisfacción. Anuario de Psicología, 39 (3), 333-350.

Cuadrado, I.; Molero, F., y Navas, M. (2003). El liderazgo de hombres y mujeres: Diferencias en estilos de liderazgo, relaciones entre estilos y predictores de variables de resultado organizacional. Revista Psicológica, 2, pp. 115-129.

Epitropaki, O. y Martin, R. (2005): "From ideal to real: A longitudinal study of the role of implicit leadership theories on leader-member exchanges and employee outcomes", Journal of Applied Psychology, 90 (4), pp. 659-676.

Lord, R.; Foti, R. y De Vader, C. (1984). A test of leadership categorization theory: Internal estructure, information processing and leadership perceptions. Organizational Behavior and Human Performance, 34, 343-378.

Lupano Perugini, M.; Blanco, E. y Castro Solano, A. (2006). Conductas de liderazgo. Una propuesta para su evaluación. XIII Jornadas de Investigación y Segundo Encuentro de Investigadores en Psicología del Mercosur.

Marky, C. (2013). La Cultura Organizacional de los Oficiales de la Región Militar del Sur del Ejército del Perú y su incidencia en el proceso de cambio institucional Año 2012. Tesis para optar el Grado Académico de Doctor en Ciencias. Arequipa: Universidad Nacional de San Agustín de Arequipa.

Martínez, M. y García, A. (1999). Comparación del perfil motivacional de un grupo de empleados en Puerto Rico: Diferencias por género y función supervisora.
Villarroel, A. (2010). Percepción del Sistema de Capacitación en las Escuelas del Ejército del Perú y su relación con el desempeño académico de los Oficiales de la Guarnición de Arequipa-2008. Tesis para optar el Grado Académico de Doctor en Ciencias. Arequipa: Universidad Nacional de San Agustín de Arequipa. 